\title{
Three-dimensional selective growth of nanoparticles on a polymer microstructure
}

\author{
Shaomin Wu ${ }^{1}$, Li-Hsin Han ${ }^{2}$ and Shaochen Chen ${ }^{2}$ \\ ${ }^{1}$ Materials Science and Engineering, The University of Texas at Austin, Austin, \\ TX 78712, USA \\ ${ }^{2}$ Mechanical Engineering Department, The University of Texas at Austin, Austin, \\ TX 78712, USA
}

Received 22 February 2009, in final form 10 May 2009

Published 23 June 2009

Online at stacks.iop.org/Nano/20/285312

\begin{abstract}
We demonstrate a new technique for selectively growing gold nanoparticles on a patterned three-dimensional (3D) polymer microstructure. The technique integrates 3D direct writing of heterogeneous microstructures with nanoparticle synthesis. A digital micromirror device is employed as a dynamic mask in the digital projection photopolymerization process to build the heterogeneous microstructure layer by layer. An amine-bearing polyelectrolyte, branched poly(ethylenimine), is selectively attached to the microstructure and acts as both a reducing and a protective agent in the nanoparticle synthesis. Scanning electron microscopy, energy dispersive $\mathrm{x}$-ray spectroscopy and $\mathrm{x}$-ray photoelectron spectroscopy are utilized to analyze the microstructure and the 3D selectivity of the nanoparticle growth.
\end{abstract}

(Some figures in this article are in colour only in the electronic version)

\begin{abstract}
Nanomaterials and nanostructures have drawn much attention due to their unique electronic, optical and chemical properties. To date, significant efforts have been devoted to the synthesis of various nanostructures such as nanowires, nanotubes, nanoshells and nanotripods [1-4]. These nanostructures have an assortment of compositions and properties, and range from elemental to compound materials and from conductors to insulators. Despite the successes in controlling the size, shape, composition distribution and state of nanomaterials, technologies for the spatial manipulation, ordered distribution or selective localization are still lacking. These problems present a major hurdle to be overcome before the proven potential of nanostructures can be applied in real applications. Significant work includes the development of optical tweezers that can manipulate nanometer-sized dielectric particles in three dimensions, by Ashkin et al and Chu [5, 6]. However, optical tweezers are limited as regards the number of nanoparticles that can be controlled for both motion and position at the same time. Different methods of aligning nanowires and nanotubes on substrates have also been demonstrated [7-9], but the ordering is only in two dimensions.

To realize three-dimensional (3D), active, heterogeneous microsystems, it is imperative to control the nanostructures in the microdevice selectively in a 3D fashion. Current microfabrication and nanofabrication techniques such as
\end{abstract}

photolithography and nanoimprinting are 2D in nature. Little work has been done on extending two-dimensional patterning to three dimensions. The capability of 3D localization of nanostructures will not only provide more functionalized area in a device, but also allow numerous $3 \mathrm{D}$ applications to be explored.

In this work, we will demonstrate the selective growth of $\mathrm{Au}$ nanoparticles in a patterned 3D polymer microstructure. Using a multi-material digital projection photopolymerization (DPP) technique [10], we fabricate a heterogeneous polymer microstructure layer by layer. We then grow Au nanoparticles in selected areas of the polymer microstructure. Materials characterization is carried out to confirm the selective decoration of the Au nanoparticles on the 3D microstructure.

As illustrated in figure 1, the DPP fabrication system includes a digital micromirror device (DMD) chip, a UV light source, a projection lens set, a height adjustable sample stage and a monomer container with feeding/purging outlets capped with a flat glass substrate. A 3D microstructure model created by computer-aided design (CAD) software was split into a sequence of cross-sectional images. These images were then reassembled by digitally forming a reflecting pattern on the micromirror array in the DMD chip. A homogenized UV light beam reaching the micromirror array was reflected and projected onto the image plane located between the glass 


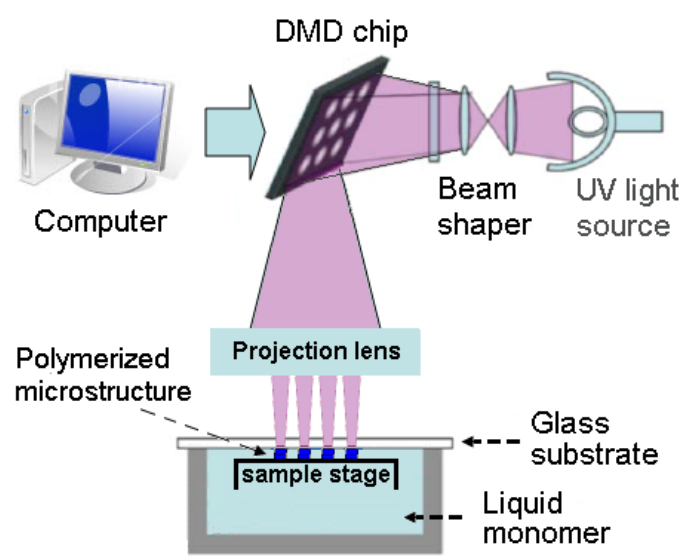

Figure 1. Schematic diagram of the DMD-based, digital projection photopolymerization (DPP) system.

substrate and the sample stage. Upon exposure to the UV light, the photo-curable liquid monomers at the image plane were polymerized and solidified, translating the cross-sectional image into a layer of the microstructure to be fabricated. If another part of the previously formed layer is to be built with a different polymer material, the liquid in the container can be switched to other kinds of monomers through the feeding/purging outlets and photopolymerized according to another corresponding pattern on the micromirror array. Once one layer of the microstructure was completed, the sample stage was lowered by an amount that is exactly equal to the thickness of the next layer to be cured. The process was repeated to stack one layer onto another until the desired 3D heterogeneous microstructure was finished.

The polymeric microstructure for demonstrating the 3D selective growth of gold nanoparticles is a two-level log-stack structure built on the basis of the multi-material DPP technique described above. As schematically illustrated in figure 2, each level of the log-stack microstructure has eight parallel micrologs, all $2 \mathrm{~mm}$ long and $50 \mu \mathrm{m}$ in width and thickness, fabricated in four steps. The first step is the polymerization of poly(ethylene glycol) diacrylate (PEGDA, Sigma-Aldrich) to define a pattern of eight parallel micrologs. In the second step, every other microlog was covered with a polymer layer cured from 1:1 methacrylic acid (MAA, Sigma-Aldrich) and pentaerythritol tetraacrylate (PTTA, Sigma-Aldrich). The remaining logs were covered with the same PEGDA material as in the first step. After rotating the pattern by $90^{\circ}$, a top level of eight logs was created with third and fourth steps which repeat the above mentioned two steps.

The microstructure was then immersed in $1 \mathrm{wt} \%$ branched poly(ethylenimine) (BPEI, Polyscience, MW: 50 000-100 000) for $4 \mathrm{~h}$. This process allows the molecular chains of the BPEI polymer to attach to the surface of MAA-PTTA micrologs due to the electrostatic interaction between the positively charged amines in the BPEI molecules and the negatively



Figure 2. Schematic of the fabrication process for the log-stack microstructure. 




Figure 3. High resolution SEM images showing the surfaces of PEGDA and MAA-PTTA micrologs on both levels.

Nanoparticle-shaped features can be observed on the surface of the MAA containing $\operatorname{logs}((\mathrm{b}),(\mathrm{d}))$ while they hardly exist on the other $\operatorname{logs}((\mathrm{a}),(\mathrm{c}))$.

charged carboxylic groups in MAA. This does not occur between the BPEI and the neutral PEGDA materials. After a thorough rinsing in DI water, the sample was left in a $0.7 \mathrm{mM} \mathrm{HAuCl}{ }_{4}$ aqueous solution at $80^{\circ} \mathrm{C}$ for $10 \mathrm{~min}$. The BPEI is known to be not only a mild reducing agent often used in the synthesis of gold or silver nanoparticles [11], but also an excellent protective agent, for avoiding aggregation of nanoparticles [12]. Once the microstructure was in the $\mathrm{HAuCl}_{4}$ solution at the elevated temperature, the $\mathrm{AuCl}_{4}^{-}$ precursors were reduced by the attached BPEI through a thermal process [13] and dispersed gold nanoparticles would form on the surface of the MAA-PTTA micrologs exclusively. It was immediately observed under a magnifying lens that the color of the MAA-PTTA micrologs on both levels turned dark red while the rest of them were unchanged. Unlike that of conventional metal materials, the color of the nanomaterials is sensitive to the shape, size and the surrounding environment of the nanostructures. This is attributed to the collective oscillations of the free electrons, often denoted as surface plasmons, on the surface of the metallic nanostructures. Dark red is one of the most frequently reported colors for gold nanoparticles [14-16]. The observation of the color change in the sample implies that gold nanoparticles, rather than a continuous gold film which would otherwise appear golden, are selectively attached to the surface of the MAA containing micrologs [17].

High resolution SEM images showing the whole log-stack microstructure and the surface of PEGDA and MAA-PTTA made micrologs in both top and bottom levels are displayed in figure 3. Nanoparticle-shaped features with sizes $\sim 50 \mathrm{~nm}$ in diameter can be recognized on the surface of the MAA containing micrologs while they hardly exist on the other logs.


$\otimes$ position of the electron probe

Figure 4. The EDS spectra taken from PEGDA and MAA-PTTA micrologs on both levels. The difference between the two spectra is that the $\mathrm{Au}$ peaks are only present in the signals from the MAA-PTTA micrologs.

SEM images of the sample were also taken after immersion in the BPEI solution but before the reduction process (not shown). The surface was smooth on the nanoscale, meaning that the nanoparticle-shaped features did not form until the reduction process was complete. To analyze the nanoscale features observed in the SEM images, EDS analysis was carried out using a JEOL JSM-5610 scanning electron microscope. The spectra were recorded by placing the electron probe $(20 \mathrm{kV})$ on the micrologs made from different materials. Comparing figures 4(a) and (b), we see that the characteristic peaks of gold are only present in the signal from the MAA-PTTA microlog. The same EDS results were also obtained from the bottom level of the microstructure. Since the EDS signal does not carry enough information about the chemical state, we also did an Xray photoelectron spectroscopy (XPS) measurement to further clarify our results. In addition to its capability of analyzing the chemical state, the XPS has a much lower detection depth $(<10 \mathrm{~nm})$ than the EDS. In this case, it is especially helpful for identifying those nanoscale features on the surface of the MAA-PTTA logs. Figure 5 shows the XPS data of the sample obtained using a Physical Electronics 5500TM XPS system with an $\mathrm{Al} \mathrm{K} \alpha$ x-ray source generating photons at an energy of $1486.6 \mathrm{eV}$. The two elemental Au signals, the $\mathrm{Au} 4 \mathrm{f}_{7 / 2}$ peak and the $\mathrm{Au} 4 \mathrm{f}_{5 / 2}$ peak, found at binding energies of $83.7 \mathrm{eV}$ and $87.5 \mathrm{eV}$ respectively, provided complementary evidence in confirming the formation of the gold nanoparticles.

A few nanoscale islands were also found on the neutral PEGDA micrologs. This is very likely due to the contamination of MAA-PTTA materials on the neutral logs. 




Figure 5. XPS data for the log-stack structure. The elemental Au peaks were found in the spectrum.

The contamination may stem from the scattering of UV light by the just-built microstructure or the small floating polymer flakes generated in the previous curing steps during the polymerization of MAA-PTTA micrologs. As a result, a small amount of BPEI was attached to the neutral logs and $\mathrm{Au}$ nanoparticles were reduced on the undesired areas. In our experiment, however, the undesired formation of gold nanoparticles on the PEGDA micrologs was below the detection sensitivity of the EDS detector. A modification of the curing process, which includes optimizing the imaging system and adjusting the concentration of reactive chemicals, will be necessary in our future work.

Since PEI can serve as both a reducing agent and a surfactant for the synthesis of many metallic nanoparticles, a wide variety of materials in nanoscale form can be localized three dimensionally on the polymer structure. Moreover, spatially selective growth of other nanostructures, such as nanowires, grown from nanoparticle seeds may also be achieved. The resolution is solely defined by the smallest feature that the DPP technique can fabricate without incorporating impurities, depending on the precision of the optical projection system. So far we have been able to achieve a linewidth of $10 \mu \mathrm{m}$ with our current setup and material recipe.

In conclusion, 3D selective growth of gold nanoparticles on a polymer microstructure with a resolution on the micrometer scale using a multi-material DPP technique was demonstrated and verified. Care must be taken when switching materials during the multi-material DPP fabrication process to achieve high selectivity. The resolution of the selective structures is limited only by the DPP process. This technique may be found applicable in the development of novel microelectromechanical systems, biosensing devices, optical materials and tissue engineering scaffolds where both nanostructures and the three-dimensional features play important roles.

\section{Acknowledgments}

This work was supported in part by research grants from the US National Science Foundation and the Office of Naval Research. We thank Texas Instruments for the donation of the DMD chip. We would also like to acknowledge Juan Zhao for her help with the EDS analysis and Tuo Wang for his help with the XPS experiment.

\section{References}

[1] von Klitzing K, Dorda G and Pepper M 1980 Phys. Rev. Lett. 45494

[2] Iijima S 1991 Nature 35456

[3] Sun Y and Xia Y 2002 Science 2982176

[4] Yan H, He R, Pham J and Yang P 2003 Adv. Mater. 15402

[5] Ashkin A and Dziedzic J M 1987 Science 2351517

[6] Chu S 1991 Science 253861

[7] Yu G, Cao A and Lieber C M 2007 Nat. Nanotechnol. 2372

[8] McAlpine M C, Ahmad H, Wang D and Heath J R 2007 Nat. Mater. 6379

[9] Huang J, Fan R, Connor S and Yang P 2007 Angew. Chem. Int. Edn. 462414

[10] Lu Y and Chen S 2008 Appl. Phys. Lett. 92041109

[11] Note C, Kosmella S and Koetz J 2006 Colloids Surf. A 290150

[12] Tan S, Erol M, Attygalle A, Du H and Sukhishvili S 2007 Langmuir 239836

[13] Sun X, Dong S and Wang E 2006 Mater. Chem. Phys. 9629

[14] Kreibig U and Vollmer M 1995 Optical Properties of Metal Clusters (Berlin: Springer)

[15] Faraday M 1857 Phil. Trans. R. Soc. 147145

[16] Eustis S and El-Sayed M A 2006 Chem. Soc. Rev. 35209

[17] Farrer R A, LaFratta C N, Li L, Praino J, Naughton M J, Saleh B E A, Teich M C and Fourkas J T 2006 J. Am. Chem. Soc. 1281796 